\title{
Parallel Numerical Simulation of Complex Unsteady Multi-Component Three-Dimensional Flow Field of Nonequilibrium Chemical Reaction
}

\author{
Liang WANG*, Ning CAI, Rui XUE, Xiaobo CUI
}

\begin{abstract}
In this paper, the gridless method, which is known for its complete independence of grids, is combined with parallel method to obtain a dynamic parallel multicomponent three-dimensional (3D) gridless method to compute the complex unsteady multi-component 3D flow field of nonequilibrium chemical reaction (NCR). Specifically, the flow field was described with a multi-component arbitrary Lagrangian-Eulerian (ALE) control equation, which contains the source term of the chemical reaction. The flow term was decoupled from the chemical reaction term, and the stiff problem of the latter term was solved by time splitting. To control the convective flux in the control equation, the multi-component artificially upstream flux vector splitting (AUFS) scheme was derived for the 3D space. In addition, 3D local point cloud reconstruction was carried out to reconstruct the abnormal point cloud near the large moving boundary in real time. Besides, geometrical zoning was adopted for the parallel part to dynamically balance the computing load across different zones. The message passing interface (MPI) was selected to realize the communication between the zones. After that, the proposed multi-component gridless algorithm was proven accurate through two examples: hydrogen combustion reaction in a vessel, and shock-induced combustion with blunt projectile. Finally, the proposed dynamic parallel multi-component 3D gridless method was applied to compute the 3D muzzle flow field of prefilled serial-connected projectiles. The evolution of the complex flow field was obtained for projectile 2. The parallel efficiency of our method surpassed $79 \%$.
\end{abstract}

Keywords: flow field of nonequilibrium chemical reaction (NCR); gridless method; moving boundary; multi-component artificially upstream flux vector splitting (AUFS) Scheme; parallel efficiency

\section{INTRODUCTION}

The difficulty in computing complex unsteady multicomponent three-dimensional (3D) chemical reaction flow lies in the complicated development of the flow field, the chemical reaction between components, and the huge computing load of grids [1-4]. In actual engineering, an unsteady flow field sometimes has complex boundaries, which are difficult and even impossible to process through meshing. The root cause is that the area or volume of grids could be negative, during the processing of complex boundaries. To solve the problem, gridless method [5], which is based on structural mechanics, becomes a new direction of the numerical simulation of the flow problem. The most attractive feature of gridless method rests with the independence of grid meshing. However, this method faces an intrinsic problem: the low computing efficiency [6]. Hence, it is very necessary to improve the expansibility and computing efficiency of the gridless method for the numerical simulation of unsteady chemical reaction flow.

The chemical reaction feature differs greatly from the flow feature in terms of time scale. As a result, the system of ordinary differential equations obtained by discretizing the control equation of unsteady chemical reaction flow is severely stiff. To overcome the stiff problem, decoupling algorithms have been increasingly adopted in recent years [7], such as Lie-Trotter splitting [8], and Strang splitting [9]. Shuen and Yoon [10] constructed the lower-upper symmetric successive overrelaxation (LU-SSOR) scheme: In the control equation, the convective term and reaction source term are computed by implicit method; the viscous dissipation term is computed by explicit method. The results show that the scheme significantly reduced the computing load. Liu et al. presented a decoupling algorithm for unsteady chemical flow, which separates effective zero-point energy or chemical enthalpy, and adds them to the source term of the chemical reaction [11-13]. By integrating adaptive structured grid method into decoupling algorithm, Ziegler et al. [14] developed a highresolution calculation program for detonation wave, and managed to capture the microstructure of detonation wave clearly. Lv and Ihme [15] defined the equivalent ratio of specific heat, and introduced discontinuous Galerkin method to simulate unsteady chemical flow. Duarte et al. [16] simulated the interaction between laminar flame and vortex structure by combining time splitting adaptive step decoupling method with spatial adaptive multi-resolution method. Gou et al. [17] proposed a dynamic multi-time scale approach, in which the unsteady chemical reaction is computed efficiently with detailed and simplified chemical reaction mechanisms. Sun et al. [18] combined dynamic multi-time scale method with dynamic adaptive chemical method into an efficient strategy called HMTS/CDAC (hybrid multi-time scale / correlated dynamic adaptive chemistry).

In computational aerodynamics, there are not many studies on gridless algorithms or their application in unsteady chemical flow. Zhuo et al. [19] integrated the upwind scheme of improved advection upstream splitting method with pressure-based weight function (AUSMPW+) into gridless algorithm, and successfully simulated the two-dimensional (2D) flow field of the perfect gas with complex quantity of heat and unsteady chemical flow field. Huh et al. [20] adopted gridless method to capture the strong shockwaves in 2D unsteady reaction flow, and found that the results agree with those of finite-volume method. Bayona et al. [21] established a discrete numerical model based on high-order gridless radial basis functiongenerated finite differences (RBF-FD), and applied to model to simulate the internal combustion of micro-rotor engine with a complex geometry.

Many scholars have demonstrated that parallel computing is an effective way to enhance the computing efficiency of gridless method and unsteady chemical reaction low [22-26]. Based on multiple instruction, multiple data (MIMD) parallel computer, Singh et al. [26] computed the problem of heat transfer and fluid flow by the element-free Galerkin (EFG) method, and achieved good speedup and efficiency. Ma et al. [27] ran a gridless 
dynamic cloud method on a graphics processing unit (GPU) with CUDA programming model [28], and simulated steady and unsteady flow fields around an airfoil. The numerical results confirm that their method can realize the speedup of more than one order of magnitude. Zhang [29] proposed a GPU-based parallel gridless method to compute Navier-Stokes equations, simulated turbulence with the Spalart-Allmaras model. In this way, they efficiently solved the flow around an airfoil. Through declarative programming, Barbosa et al. [30] defined a physical mathematical model, and realized a novel parallel strategy for gridless method. Numerical results show that the strategy is accurate and competitive. However, none of the above writings on parallel computing tackles unsteady chemical reaction.

\section{CONTROL EQUATION AND NUMERICAL METHOD}

In the system of rectangular coordinates, the ALE control equation containing the source term of the chemical reaction can be expressed as:

$\boldsymbol{Q}_{t}+\boldsymbol{E}_{1, x}+\boldsymbol{E}_{2, y}+\boldsymbol{E}_{3, z}=\boldsymbol{R}$

where, $\boldsymbol{Q}$ is a conserved variable; $\boldsymbol{E}_{1}, \boldsymbol{E}_{2}$ and $\boldsymbol{E}_{3}$ are convective fluxes; $x, y$ and $z$ are subscripts representing partial derivation; $\boldsymbol{R}$ is the source term of the chemical reaction. The specific forms of these variables are as follows:

$$
\begin{aligned}
& \boldsymbol{Q}=\left(\begin{array}{llllllll}
\xi_{1} & \xi_{1} & \ldots & \xi_{N_{R}} & \xi_{u_{1}} & \xi_{u_{2}} & \xi_{u_{3}} & \xi E
\end{array}\right)^{\mathrm{T}} \\
& \boldsymbol{R}=\left(\begin{array}{llllllll}
\kappa_{1} & \kappa_{1} & \ldots & \kappa_{N_{R}} & 0 & 0 & 0 & 0
\end{array}\right)^{\mathrm{T}} \\
& \boldsymbol{E}_{1}=\left(\begin{array}{c}
\xi_{1} u_{1}^{\prime} \\
\xi_{2} u_{1}^{\prime} \\
\vdots \\
\xi_{N_{R}} u_{1}^{\prime} \\
\xi u_{1} u_{1}^{\prime}+p \\
\xi u_{2} u_{1}^{\prime} \\
\xi u_{3} u_{1}^{\prime} \\
\xi E u_{1}^{\prime}+u_{1} p
\end{array}\right) ; \boldsymbol{E}_{1}=\left(\begin{array}{c}
\xi_{1} u_{2}^{\prime} \\
\xi_{2} u_{2}^{\prime} \\
\vdots \\
\xi_{N_{R}} u_{2}^{\prime} \\
\xi u_{1} u_{2}^{\prime}+p \\
\xi u_{2} u_{2}^{\prime} \\
\xi u_{3} u_{2}^{\prime} \\
\xi E u_{2}^{\prime}+u_{2} p
\end{array}\right) ; \boldsymbol{E}_{1}=\left(\begin{array}{c}
\xi_{1} u_{3}^{\prime} \\
\xi_{2} u_{3}^{\prime} \\
\vdots \\
\xi_{N_{R}} u_{3}^{\prime} \\
\xi u_{1} u_{3}^{\prime}+p \\
\xi u_{2} u_{3}^{\prime} \\
\xi u_{3} u_{3}^{\prime} \\
\xi E u_{3}^{\prime}+u_{3} p
\end{array}\right) \\
& u_{1}^{\prime}=u_{1}-w_{1}, u_{2}^{\prime}=u_{2}-w_{2}, u_{3}^{\prime}=u_{3}-w_{3}
\end{aligned}
$$

where, $\xi_{i}$ is the mass density of component $i ; N_{R}$ is the total number of components; $\xi$ is the mass density of the mixed gas; $u_{1}, u_{2}$ and $u_{3}$ are the velocity components of the mixed gas in the $\mathrm{x}, \mathrm{y}$ and $\mathrm{z}$ directions, respectively; $w_{1}, w_{2}$ and $w_{3}$ are the velocity components of a grid node in the $x, y$ and $z$ directions, respectively; $p$ is the pressure of the mixed gas; $\xi E$ is the total energy per unit volume; $\kappa_{i}$ is the mass generation rate of component $i$ induced by the chemical reaction:

$$
\begin{aligned}
& \kappa_{i}=\sum_{t=1}^{R_{N}}\left(v_{i t}^{R}-v_{i t}^{L}\right)\left[K_{f t} \prod_{k=1}^{N_{R}}\left(\frac{\xi_{k}}{M_{k}}\right) v_{k t}^{L}-\right. \\
& \left.-K_{b t} \prod_{k=1}^{N_{R}}\left(\frac{\xi_{k}}{M_{k}}\right) v_{k t}^{R}\right] \cdot M_{i}
\end{aligned}
$$

where, $v_{i t}^{L}$ and $v_{i t}^{R}$ are the equivalence coefficients on the left and right sides of chemical reaction t, respectively; $M_{i}$ is the molar mass of component $i ; R_{N}$ is the total number of chemical reactions in the model; $K_{f t}$ is the forward and reverse reaction rate constant, which can be calculated by the Arrhenius equation:

$$
K_{f t}=A_{t} T^{b_{t}} \exp \left(-\frac{E_{t}}{R_{u} T}\right)
$$

where, $A_{t}$ is the pre-exponential factor; $b_{t}$ is the temperature factor; $E_{t}$ is the activation energy; $R_{u}$ is the universal gas constant; $T$ is the temperature of the mixed gas. The reverse reaction rate constant can be derived from the reaction equilibrium constant, while the source term of the chemical reaction can be processed by the finite rate reaction model.

Each component and mixed gas satisfies the equation of state:

$p=\xi K T$

where, $K$ is the gas constant of the gas.

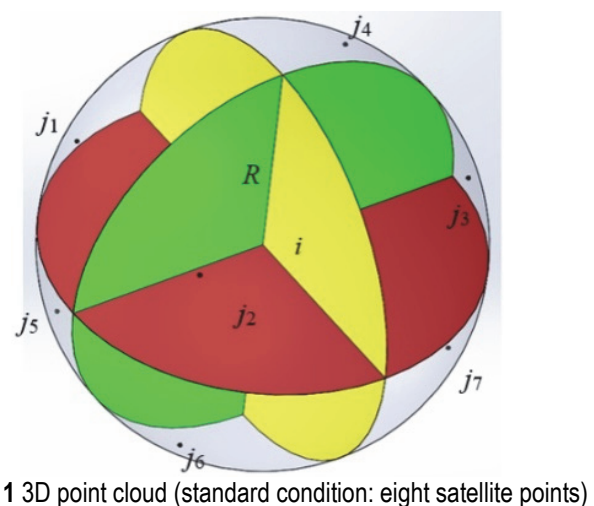

Figure $13 \mathrm{D}$ point cloud (standard condition: eight satellite points)

Let $(x, y, z, f)$ be the unique coordinates of a point in the entire physical space, with $\mathrm{f}$ being the flow function value of that point. In the point cloud of point $i$ (Fig. 1), function $\mathrm{f}$ can be described as a one-order linear function:

$f=a x+b y+c z+d$

Let $N$ be the number of satellite points in Fig. 1. The center point and each point in the point cloud should satisfy the above linear function. Through simple transformation, Eq. (3) can be changed into:

$$
\left\{\begin{array}{l}
f_{i 1}=a x_{i 1}+b y_{i 1}+c z_{i 1}+1 \\
f_{i N}=a x_{i N}+b y_{i N}+c z_{i N}+1
\end{array}\right.
$$


where,

$x_{i s}=\frac{x_{s}+x_{i}}{2}, y_{i s}=\frac{y_{s}+y_{i}}{2}, z_{i s}=\frac{z_{s}+z_{i}}{2}$ and $f_{i s}=\frac{f_{s}+f_{i}}{2}$, with $s$ being the $s$-th satellite point. Since the number of satellite points in the point cloud $N>3$, the above formula is a contradictory set of equations. Suppose $E=\sum_{s=1}^{N}\left[\Delta f_{i s}-\left(a \Delta x_{i s}+b \Delta y_{i s}+c \Delta z_{i s}\right)\right]^{2}$. The three parameters $a, b$ and $c$ should minimize $E$, such that the fitted surface approximates the physical solution. Hence, $\frac{\partial E}{\partial a}=\frac{\partial E}{\partial b}=\frac{\partial E}{\partial c}=0$. Let $M$ be the coefficient matrix of the above set of equations. Then, we have $a=\sum_{s=1}^{N} \lambda_{i s}^{2} \frac{f_{i}+f_{s}}{2} \quad, \quad b=\sum_{s=1}^{N} \lambda_{i s}^{2} \frac{f_{i}+f_{s}}{2} \quad, \quad$ and $c=\sum_{s=1}^{N} \lambda_{i s}^{2} \frac{f_{i}+f_{s}}{2}$, where coefficient $\lambda_{i s}^{j}(j=1,2,3)$ is an element in the $j$-th row of the new matrix $\left(\boldsymbol{M}^{\boldsymbol{T}} \boldsymbol{M}\right)^{-1} \boldsymbol{M}^{\boldsymbol{T}}$. Thus, the flux at any point $\mathrm{i}$ in the flow field can be solved by

$$
\left.\frac{\partial \boldsymbol{E}_{1}}{\partial x}\right|_{i}+\left.\frac{\partial \boldsymbol{E}_{2}}{\partial y}\right|_{i}+\left.\frac{\partial \boldsymbol{E}_{3}}{\partial z}\right|_{i}=\sum_{j=1}^{N}\left(\sqrt{\left(\lambda_{i s}^{1}\right)^{2}+\left(\lambda_{i s}^{2}\right)^{2}+\left(\lambda_{i s}^{3}\right)^{2} \boldsymbol{F}_{i j}}\right)
$$

The artificially upstream flux vector splitting (AUFS) [31] is a simple yet highly robust scheme that accurately recognizes contact discontinuities, and avoids carbuncle instability in the case of multidimensionality. The 3D multicomponent AUFS scheme can be expressed as:

$F_{i j}^{\mathrm{AUFS}}=(1-M) \boldsymbol{F}_{1}+M \boldsymbol{F}_{2}$

where, $\boldsymbol{F}_{1}$ and $\boldsymbol{F}_{2}$, are fluid vectors; $M$ is a constant:

$$
\begin{aligned}
& \boldsymbol{F}_{1}=\left[\frac{1}{2}\left(\boldsymbol{P}_{i}+\boldsymbol{P}_{j}\right)+\delta \boldsymbol{U}\right], \boldsymbol{F}_{2}=\left[\boldsymbol { U } ^ { \alpha } \left(\tilde{\left.\left.u^{\alpha}-w^{\alpha}-S_{2}\right)+\boldsymbol{P}^{\alpha}\right],}\right.\right. \\
& M=S_{1} /\left(S_{1}-S_{2}\right)
\end{aligned}
$$

where, $P$ is the pressure component; $\delta U$ is the artificial viscosity term:

$$
\begin{aligned}
& \tilde{u^{\alpha}}=n_{x} u_{1}^{\alpha}+n_{y} u_{2}^{\alpha}+n_{z} u_{3}^{\alpha} ; \tilde{w}^{\alpha}=n_{x} w_{1}^{\alpha}+n_{y} w_{2}^{\alpha}+n_{z} w_{3}^{\alpha}, \\
& \boldsymbol{P}=\left(0,0, \ldots, 0, \mathrm{p} n_{x}, p n_{y}, p n_{z}, p \tilde{u}\right)^{\mathrm{T}} \\
& n_{x}=b_{i j} / e_{i j}, n_{y}=c_{i j} / e_{i j}, n_{z}=c_{i j} / e_{i j}, e_{i j}=\sqrt{b_{i j}^{2}+c_{i j}^{2}+d_{i j}^{2}} \\
& \delta \boldsymbol{U}=\frac{1}{2 c^{j}}\left(\left(Y_{1} p\right)_{i}\left(Y_{2} p\right)_{i} \ldots\left(Y_{N_{R}} p\right)_{i}\left(p u_{1}\right)_{i}\left(p u_{2}\right)_{i}\left(p u_{3}\right)_{i}\right. \\
& \left.(H p)_{i}\right)^{\mathrm{T}}-\frac{1}{2 c^{j}}\left(\left(Y_{1} p\right)_{j}\left(Y_{2} p\right)_{j} \ldots\left(Y_{N_{R}} p\right)_{j}\left(p u_{1}\right)_{j}\left(p u_{2}\right)_{j}\right. \\
& \left.\left(p u_{3}\right)_{j}(H p)_{j}\right)^{\mathrm{T}}
\end{aligned}
$$

where, $Y_{i}$ is the mass fraction of component $i, Y_{i}=\xi_{i} / \xi$; $\alpha$, coefficient $M$, and scale functions $S_{1}$ and $S_{2}$ can be calculated by:

$\alpha=\left\{\begin{array}{l}i S_{1}>0 \\ j S_{1} \leq 0\end{array}\right.$,

$M=S_{1} /\left(S_{1}-S_{2}\right), S_{1}=\left(u_{1}^{i}+u_{1}^{j}\right) / 2$,

$S_{2}=\left\{\begin{array}{l}\operatorname{Min}\left(0, u_{1}^{i}-w_{1}-c^{i}, u^{*}-c^{*}\right) S_{1}>0 \\ \operatorname{Max}\left(0, u_{1}^{j}-w_{1}-c^{j}, u^{*}-c^{*}\right) S_{1} \leq 0\end{array}\right.$

$\alpha=\left\{\begin{array}{l}i S_{1}>0 \\ j S_{1} \leq 0\end{array}\right.$,

where, $u^{*}$ and $c^{*}$ are intermediate wave velocity and intermediate sound velocity derived by isentropic relationship:

$$
u^{*}=\frac{\left(u_{1}^{i}+u_{1}^{j}\right)}{2}+\frac{c^{i}-c^{j}}{\gamma-1}, c^{*}=\frac{\left(c^{i}+c^{j}\right)}{2}+\frac{(\gamma-1)\left(u_{1}^{i}-u_{1}^{j}\right)}{4}
$$

where, $\gamma$ is the ratio of specific heat of the gas.

To handle the coupling between the terms in the control equation of multi-component chemical reaction, the most suitable operators can be selected by time splitting for different physical processes. In principle, the time step could be the shortest among the allowable time steps for all physical processes. The specific process is as follows: freeze the chemical reaction, and compute flow field parameters; treat the chemical reaction as a constant volume exothermic process, and conduct multi-step calculation until the total time interval reaches $\Delta t_{\text {fluid. }}$. Then, the Runge-Kutta methods were adopted to solve the ordinary differential equations by marching a small step at a time.

The semi-discrete form of the governing equation in this paper can be written as:

$\boldsymbol{Q}_{i}+\boldsymbol{L}_{i}=0$ is:

The general form of the $m$-step Runge-Kutta method

$\boldsymbol{Q}_{i}^{0}=\boldsymbol{Q}_{i}^{N}$

$\boldsymbol{Q}_{i}^{1}=\boldsymbol{Q}_{i}^{0}-\alpha_{1} \boldsymbol{L}_{i}\left(\boldsymbol{Q}_{i}^{0}\right) \Delta t$

$\boldsymbol{Q}_{i}^{2}=\boldsymbol{Q}_{i}^{0}-\alpha_{2} \boldsymbol{L}_{i}\left(\boldsymbol{Q}_{i}^{1}\right) \Delta t$

$\vdots=\vdots$

$\boldsymbol{Q}_{i}^{m}=\boldsymbol{Q}_{i}^{0}-\alpha_{m} \boldsymbol{L}_{i}\left(\boldsymbol{Q}_{i}^{m-1}\right) \Delta t$

$\boldsymbol{Q}_{i}^{N+1}=\boldsymbol{Q}_{i}^{m}$

$\alpha_{k}=\frac{1}{m-k+1}, k=1, \ldots, m$

In this paper, the flow field is simulated numerically by gridless method, which involves rigid walls and farfield boundary conditions. The flow on rigid walls satisfies 
the impermeability condition, while the far-field boundary conditions aim to reduce the influence of the finite computational domain on flow field simulation, i.e., to eliminate artificial boundary layer and artificial boundary reflection. Both were handled by setting up mirrored virtual points.

\section{DYNAMIC POINT CLOUD PROCESSING NEAR MOVING BOUNDARY}

The large movement of the boundary could reduce the quality of the point cloud near the boundary. The resulting low-quality abnormal point cloud must be reconstructed before calculation. The gridless weighted point filling method can be understood as throwing lots of beans into the computational domain. Each bean has two attributes, namely, the position and radius of the center point, respectively. The former is the coordinates of the gridless node, and the latter is the weight of that node. The size of the beans may vary. Small beans can be placed in the parts of the flow field, which need to be densified; large beans can be placed in the parts, which need to be sparse, as shown in Fig. 2.

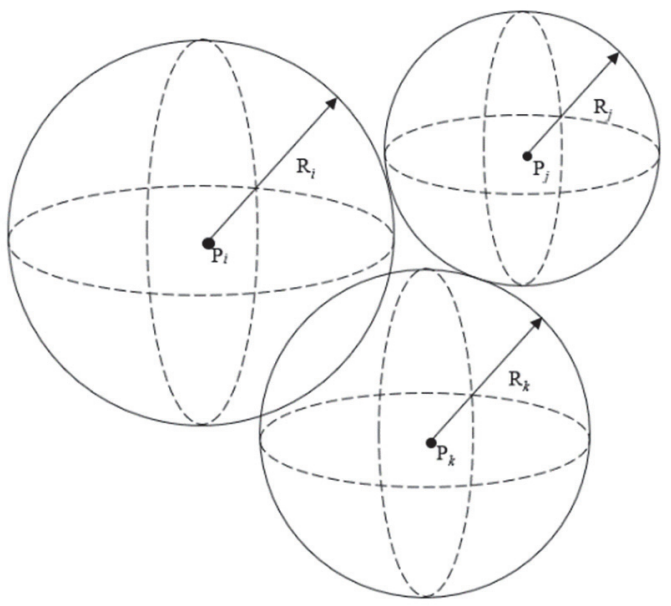

Figure 2 Point weight and point distribution diagram

During the filling process, this paper looks for each new bean with advancing front technique. In Fig. 3, the triangle $\mathrm{ABC}$ is the initial advancing front, and the position $\left(x_{i}, y_{i}, z_{i}\right)$ and radius $r_{i}$ of the new advancing point $\mathrm{D}$ can be calculated by:

$$
\left\{\begin{array}{l}
\left(x_{1}-x_{i}\right)^{2}+\left(y_{1}-y_{i}\right)^{2}+\left(z_{1}-z_{i}\right)^{2}=\left(r_{1}-r_{i}\right)^{2} \\
\left(x_{2}-x_{i}\right)^{2}+\left(y_{2}-y_{i}\right)^{2}+\left(z_{2}-z_{i}\right)^{2}=\left(r_{2}-r_{i}\right)^{2} \\
\left(x_{3}-x_{i}\right)^{2}+\left(y_{3}-y_{i}\right)^{2}+\left(z_{3}-z_{i}\right)^{2}=\left(r_{3}-r_{i}\right)^{2} \\
r_{i}=\frac{1}{3} \cdot\left(r_{1}+r_{2}+r_{3}\right) \cdot \beta_{r}
\end{array}\right.
$$

where, the subscripts $1-3$ represent the 3 known adjacent beans $A, B$ and $C ; \beta_{r}$ is the filling coefficient. The adjacent beans are allowed to be nontangential or elastic.

The dynamic point cloud processing is realized in the following steps: (1) deleting abnormal point cloud to form a reconstructed cavity surface, as shown in Fig. 4; (2) filling new nodes by weighted point filling method, and updating the point cloud; (3) optimizing the point cloud through Laplacian smoothing and surface-line conversion.
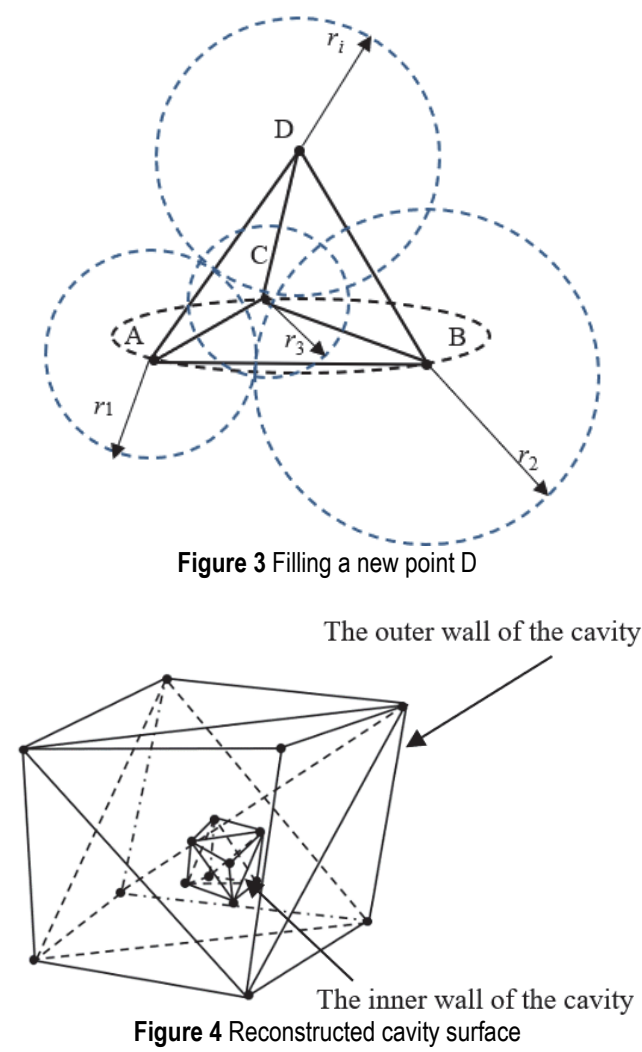

\section{DESIGN OF PARALLEL ALGORITHM}

Parallel computing, an effective way to improve computing efficiency, promotes the application of gridless method in actual engineering. The efficiency of parallel computing is jointly determined by the level of parallelism, computing granularity, time and space complexity, communication delay, scheduling strategy, and load balancing. To properly handle these factors and their relationship and fully utilize parallelism, it is necessary to design parallel algorithm and program. The high efficiency of the parallel computer lies in its unique structure. The efficiency of the parallel machine cannot be fully untapped, unless efficient design parallel algorithm and program are designed to match the hardware parallelism, in the light of the structural features.

This paper improves the gridless method and explores deep into the parallel algorithm, trying to numerically simulate the 3D complex flow field by gridless method. The specific steps include parallel search of satellite points, load balancing, etc. Fig. 5 shows the information transfer process of adjacent computational zones.

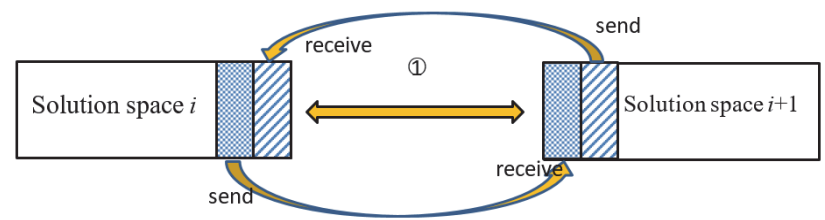

, 1 : Message passing interface (MPI) is adopted for information transfer between adjacent zones

Figure 5 Information transfer between adjacent computational zones 


\subsection{Segmentation of Computational Domain}

The computational domain needs to be segmented without disrupting the load balance between processes, which is the key to improving the efficiency of parallel computing. There are two criteria for the segmentation: (1) The segmented zones should have basically the same number of discrete points; (2) The boundary length of each zone should be minimized. The former criterion ensures load balancing; the latter determines the communication time. The former is the basis of the latter. These two criteria lay the foundation for evaluating the segmentation on algorithm performance.

The gridless method only involves the discrete points distributed in space, without any information about grid units. Therefore, the 3D computational domain tends to be a regular rectangle, cylinder, or the combination of the two shapes. If the discrete points are evenly distributed across the computational domain, the domain can be manually divided by plane segmentation into regular discrete zones, according to the shape of the domain (Fig. 6). Considering the presence of the significant moving boundary in the domain, this paper adopts the dynamic zoning approach, which assigns a unique computing load coefficient to each node. For instance, a node on the moving boundary was assigned a coefficient greater than 1 . When the moving boundary travels from one solution space to the other, the boundaries between the zones for parallel computing must be adjusted to balance the computing load across different zones.

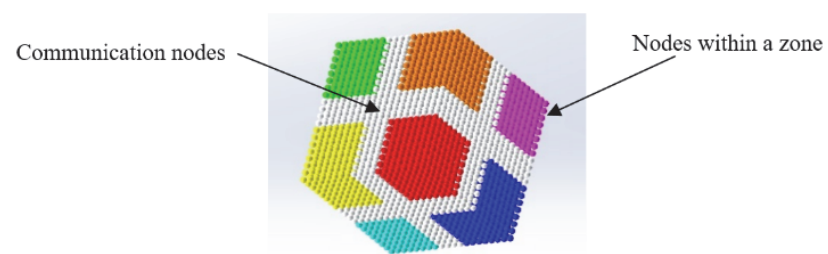

Figure 6 Zoning (white nodes are communication nodes; the eight colours stand for the eight zones)

\subsection{Flow of Parallel Computing}

Fig. 7 shows the process of our parallel computing approach, which includes task initiation, ow field zoning and computing, reconstruction of low-quality point clouds, information exchange and update between adjacent zones, redistribution of computing load, and outputting flow field data after the termination condition is met through multiple iterations.

\subsection{Search Efficiency of Satellite Points in Parallel Computing}

In the gridless method, the search of satellite points consumes lots of machine-hours. During serial computing, zone-based search was adopted to speed up the query for satellite points. Suppose the computational domain contains $\mathrm{N}$ computing nodes, and the discrete space is divided into $\mathrm{m}$ zones. Then, the single-process search for satellite points needs $\frac{N}{m} \times\left(\frac{N}{m}-1\right)$ computations. In theory, the zone-based search of parallel computing only consumes $1 / \mathrm{m}^{2}$ of the original computing time.

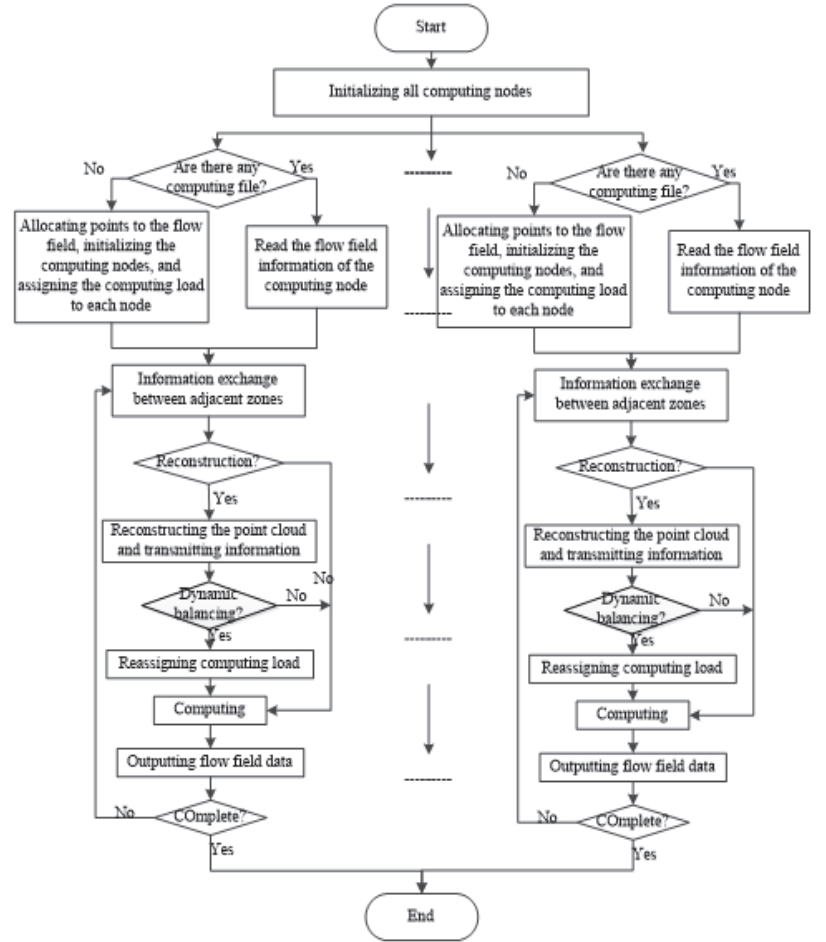

Figure7 Parallel computing process

To disclose the trend of the parallel search efficiency with the changing number of processes, 8 million points were evenly arranged in the $3 \mathrm{D}$ space $(200 \times 200 \times 200)$, and a parallel search was carried out in the peer-to-peer mode with $1,2,4,6,8,10$, or 12 processes. Fig. 8 compares the speedup and parallel efficiency in different settings. The results show that the gridless method achieved good parallelism and parallel efficiency in the search of satellite points, thanks to its unique search method. The more the processes, the greater the speedup, and the higher the parallel efficiency.

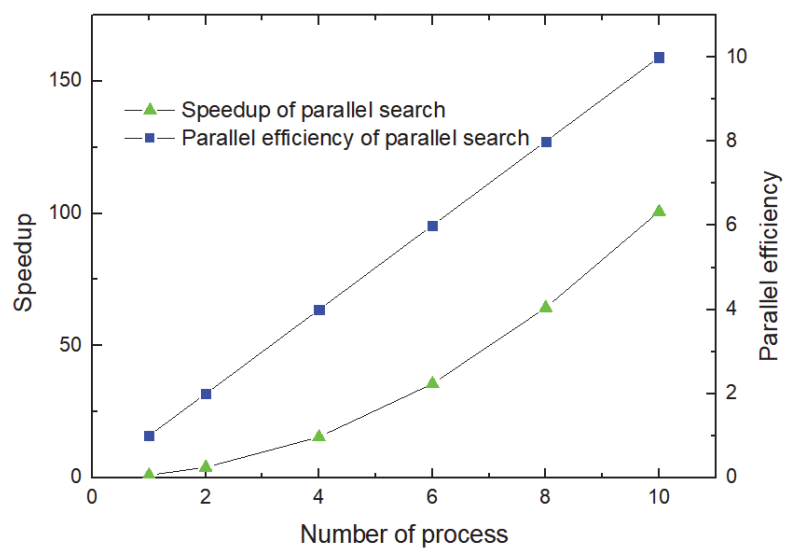

Figure 8 Search efficiency of satellite point search of parallel computing

\section{Numerical Calculations}

\subsection{Hydrogen Combustion Reaction in a Constant Volume Vessel}

In a constant volume vessel, the mixture of $\mathrm{H}_{2}$ and $\mathrm{O}_{2}$ (equivalence ratio $=1: 1$ ) was ignited. At time $t=0$, the temperature and pressure in the vessel were set to $1,600 \mathrm{~K}$ and 101,325.0 Pa (barometric pressure), respectively. The stochastic reaction model of 9 components and 17 reactions 
was adopted for the vessel. By simulating the combustion reaction, the research team attempted to verify the correctness of the proposed dynamic solver for multicomponent reaction.

Fig. 9 shows the time variation curves of the combustion temperature and pressure of $\mathrm{H}_{2}$ and $\mathrm{O}_{2}$ in the constant volume vessel, and that of their combustion products. The dotted lines are the equilibrium combustion components of $\mathrm{H}_{2}$ and $\mathrm{O}_{2}$ derived by thermodynamics

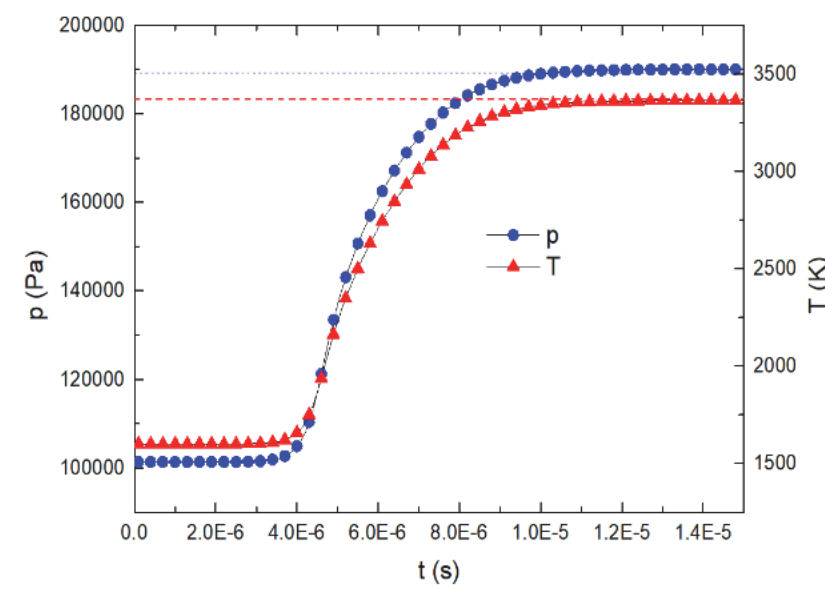

(a) Time variation of temperature and pressure theory. It can be observed that the error was within $7 \%$, reflecting the validity of our numerical results: under the equilibrium state, the pressure intensity was $189,994.3 \mathrm{~Pa}$, and temperature was $3,363.3 \mathrm{~K}$. Judging by the trend of the curves, the reaction was slow in the early phase, due to the low contents of $\mathrm{H}, \mathrm{O}$, and $\mathrm{OH}$ free radicals; the reaction speed suddenly picked up, as the free radicals accumulated to certain contents, and eventually arrived at the thermodynamic equilibrium state.

Figure 9 Combustion of $\mathrm{H}_{2}$ and $\mathrm{O}_{2}$ in the constant volume vessel

\subsection{Shock-Induced Combustion with Blunt Projectile}

In a premixed air of hydrogen and the air (equivalence ratio $=1: 1$ ), when the moving velocity of a blunt projectile is greater than the Chapman-Jouguet (CJ) detonation velocity, the combustion front will couple with the shockwave front, while the entire flow field is stable, i.e., in the overdriven detonation state. This paper numerically computes this state by $3 \mathrm{D}$ gridless algorithm. The premixed air of hydrogen and the air (equivalence ratio $=$ 1:1) was entered via the inlet of the flow field, with a pressure of $42,662 \mathrm{~Pa}$, a temperature of $250 \mathrm{~K}$, and a velocity of $2,605 \mathrm{~m} / \mathrm{s}$. The head diameter of the blunt projectile was set to $15 \mathrm{~mm}$. A total of $1,564,236$ points were arranged in the flow field. In view of the flow field features, the head part of the projectile was meshed into denser grids than other parts.

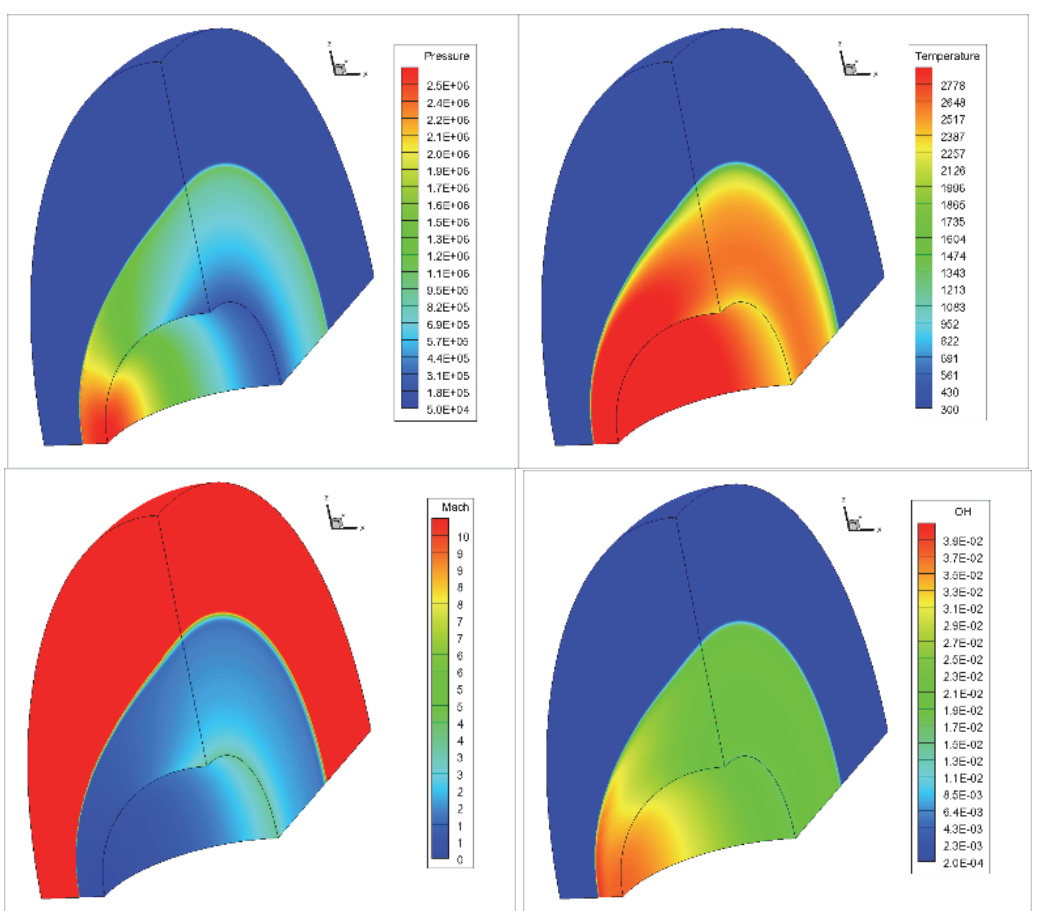

Figure $10 \mathrm{Cloud} \mathrm{maps} \mathrm{of} \mathrm{the} \mathrm{pressure,} \mathrm{temperature,} \mathrm{Ma}$, and $\mathrm{OH}$ distributions for shock-induced combustion with blunt projectile 
Fig. 10 presents the cloud maps of the pressure, temperature, $\mathrm{Ma}$, and $\mathrm{OH}$ distributions for shock-induced combustion with blunt projectile. The fast incoming flow formed a curved detached shockwave in front of the projectile. After the mixed gas passed through the shockwave, its velocity dropped below the velocity of sound. The falling velocity was accompanied with rising temperature and pressure. As a result, the mixed gas was completly combusted in a very short period, the induction segment was almost invisible, and the flame front coincided with the shockwave. Tab. 1 compares the stagnation point pressure and temperature in the front of the blunt projectile, and the shockwave position with those of theory [32]. The two sets of values agree well with each other.

Table 1 Comparison between the stagnation point pressure and temperature in the front of the blunt projectile, and the shockwave position with those of theory

\begin{tabular}{|c|c|c|c|}
\hline & $\mathrm{T}$ & $\mathrm{P}$ & Shockwave position \\
\hline Ref. [32] & 3,576 & 2,623 & $1.200 \mathrm{R}$ \\
\hline Our results & 3,513 & 2,613 & $1.191 \mathrm{R}$ \\
\hline
\end{tabular}

\subsection{D Muzzle Flow Field of Prefilled Serial-Connected Projectiles}

When a machine gun shoots at a fast speed, the next projectile exits the muzzle before the dispersion of the muzzle flow field of the current projectile, creating a complex highly under-expanded gas jet. It is assumed that two identical projectiles are loaded in the barrel, and the second projectile starts moving when the first projectile reaches a distance to the right side of the muzzle, which is 5 times the barrel diameter. The computational domain of the muzzle is symmetrical about the rotting axis. Figy. 11 illustrates the computational domain on the section XOY. At time $t=0$, both projectiles 1 and 2 are to the left of the muzzle. The barrel length was set to $1.08 \mathrm{~m}$; the flow field out of the muzzle was designed as a cylinder with a radius of $0.5 \mathrm{~m}$ and a length of $2.2 \mathrm{~m}$. Due to the symmetry of the flow field, only $1 / 4$ of the cylinder was selected for computation. Considering the features of the multicomponent muzzle flow field, a total of 5,575,322 points were deployed in the flow field of this example. Then, the 3D parallel gridless algorithm was introduced to compute the 3D muzzle flow field of the serial-connected projectiles.

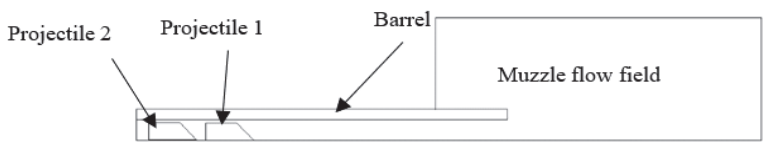

Figure 11 Computational domain of muzzle
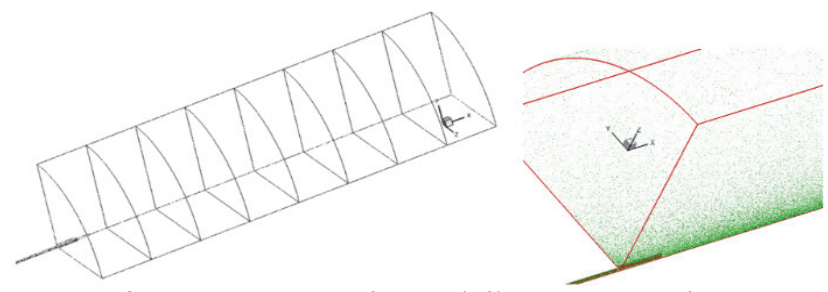

Figure 12 Computational domain of muzzle (left) and distribution of some nodes (right)

Fig. 12 shows the flow field at the initial moment. The computational domain was divided into eight zones. Thus,
8 computer processes were called for calculation. During the computing process, the computing load in each zone was adjusted timely, as the projectiles moved from one zone to another.

Fig. 13 displays the cloud map of temperature on $y=0$ section and that of $\mathrm{OH}$ distribution on $z=0$ section in the 3D muzzle flow field of prefilled serial-connected projectiles at $t=4.54 \mathrm{~ms}, t=4.65 \mathrm{~ms}, t=4.83 \mathrm{~ms}, t=5.11$ $\mathrm{ms}, t=5.25 \mathrm{~ms}$, and $t=5.33 \mathrm{~ms}$. OH is an intermediate product of the chemical reaction between $\mathrm{H}_{2}$ and $\mathrm{O}_{2}$ in gunpowder. It can be used to determine the position of the chemical reaction at the muzzle. The reaction intensity is positively correlated with the $\mathrm{OH}$ content. Fig. 14 displays the cloud map of density on $y=0$ section and that of pressure distribution on $z=0$ section in the 3D muzzle flow field of projectile 2 at the same moments. The figure vividly depicts the complex jetting of the highly underexpanded gas produced by gunpowder combustion in the muzzle of the 3D prefilled machine gun. The wave system was much more complex than that of a single projectile. When projectile 2 flew away from the muzzle, the flow field at the muzzle was the bottle-shaped shockwave system produced by the highly under-expanded gunpowder gas. As shown in Fig. 13a, projectile 2 entered the shockwave bottle. Meanwhile, the highly under-expanded gunpowder gas, which belonged to projectile 2 , in the barrel expanded rapidly. The jetting velocity changed suddenly to surpass the projectile velocity. The jet quickly damaged the shockwave bottle, rushed into the muzzle flow field, and propagated in the axial and radial directions. In this way, the combustible $\mathrm{H}_{2}$ and $\mathrm{CO}_{2}$ in the flow field were supplemented. In the oxygen-rich hot area of the flow field, the chemical reaction became dramatic again, and the mass fraction of $\mathrm{OH}$ also revived. The flame propagated reversely to the muzzle area (Fig. $13 \mathrm{~b}$ to Fig. 13d), pushing up the temperature near the muzzle once more. In the meantime, the high-energy gas entering the flow field strengthened the weakening entrainment effect, increased the vortex rings, and absorbed more air. As a result, the chemical reaction became more violent at the edge of the flow field, the $\mathrm{OH}$ mass fraction rose, and the flame spread to the peripherals of the flow field. Due to the supplement of new energy, the shockwave at the muzzle gradually evolved into a larger crown-shaped shockwave. The secondary flame spread further away from the muzzle, but the shockwave bottle disappeared (Fig. 14). With the continuous evolution of the flow field, the velocity of the gunpowder gas slowed down. The gas movement speed was surpassed by projectile 2. As the projectile moved away from the muzzle flow field, a shockwave formed at the heat of the projectile.

Fig. 15 presents the cloud map of the temperature of projectile 2 on the YOZ section at $30 D$ ( $D$ refers to barrel diameter) from the right side of the muzzle. The temperature variation shows that the combustion area firstly shrunk and then expanded. The shrinking is probably the result of forward propagation of the combustible gas, and the expansion is attributable to the reverse propagation of the flame along the axial direction, owing to the supplementation of new combustible gas. The flame also expanded outward along the radial direction, for new air was entrained by the enhanced vortex rings on the edge of the flow field. Fig. 16 presents the distribution of 
pressure intensity on the surface of projectile 2 at $t=4.54$ $\mathrm{ms}, t=4.65 \mathrm{~ms}, t=4.83 \mathrm{~ms}, t=5.11 \mathrm{~ms}, t=5.25 \mathrm{~ms}$, and $t=5.33 \mathrm{~ms}$. Fig. 17 presents the variation curves of pressure and temperature along the $x$ direction on the surface of projectile 2 at the muzzle flow field. The pressure and temperature on the surface of the projectile were on the rise. The pressure intensity was relatively high, as the projectile moved through the flow field of gunpowder gas.

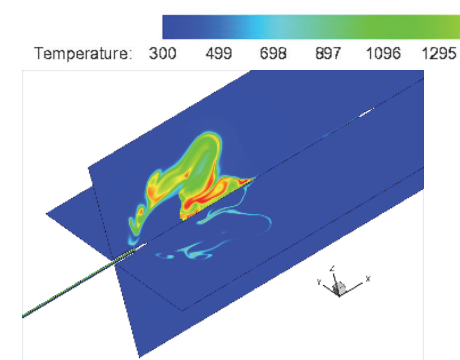

(a) $t=4.54 \mathrm{~ms}$

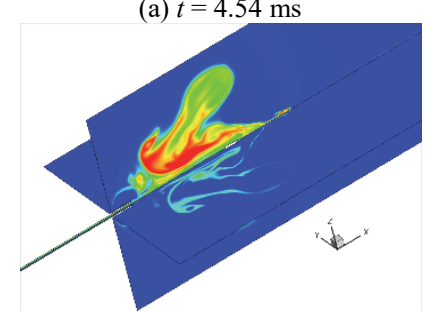

(d) $t=5.11 \mathrm{~ms}$

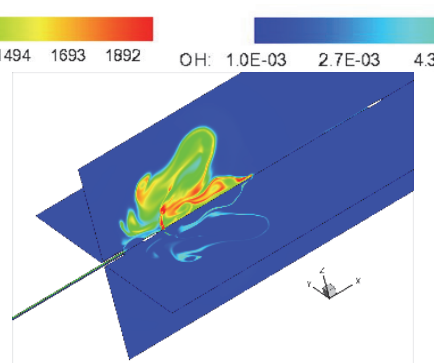

(b) $t=4.65 \mathrm{~ms}$

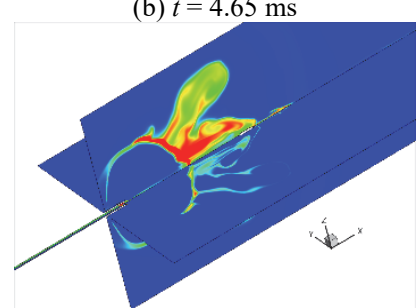

(f) $t=5.25 \mathrm{~ms}$

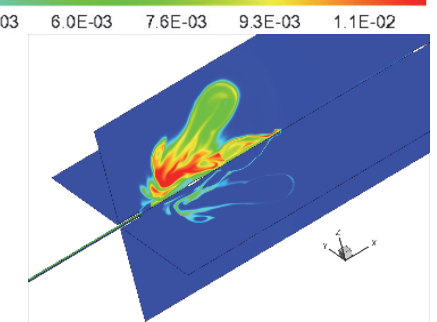

(c) $t=4.83 \mathrm{~ms}$

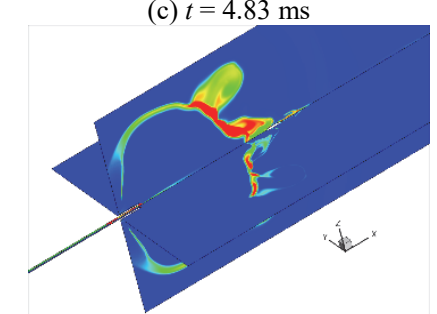

(g) $t=5.33 \mathrm{~ms}$

Figure 13 Cloud map of temperature on $y=0$ section and that of $\mathrm{OH}$ distribution on $z=0$ section in the 3D muzzle flow field of prefilled serial-connected projectiles

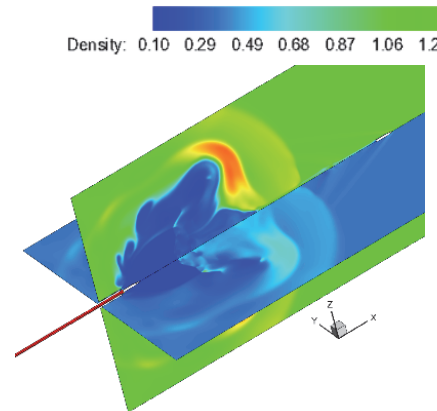

(a) $t=4.54 \mathrm{~ms}$

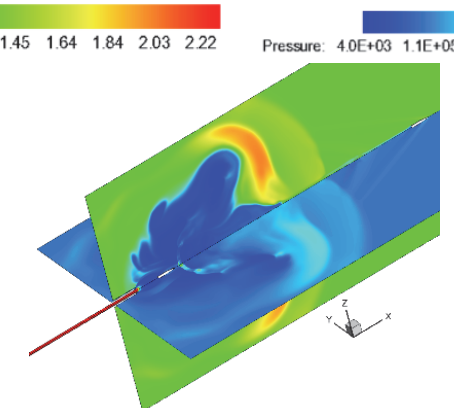

(b) $t=4.65 \mathrm{~ms}$

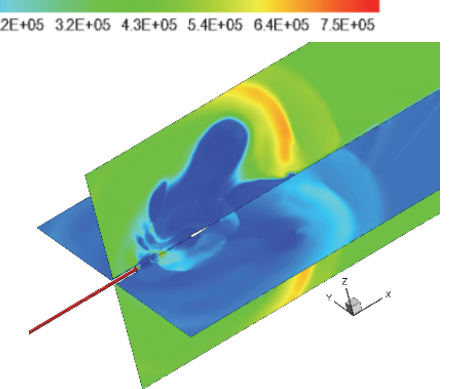

(c) $t=4.83 \mathrm{~ms}$

Figure 14 Cloud map of density on $y=0$ section and that of pressure distribution on $z=0$ section in the 3D muzzle flow field of projectile 2

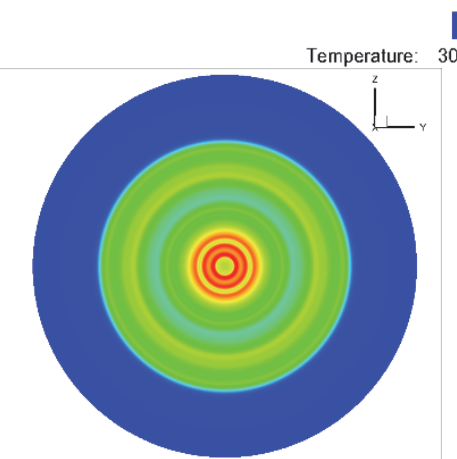

(a) $t=4.54 \mathrm{~ms}$

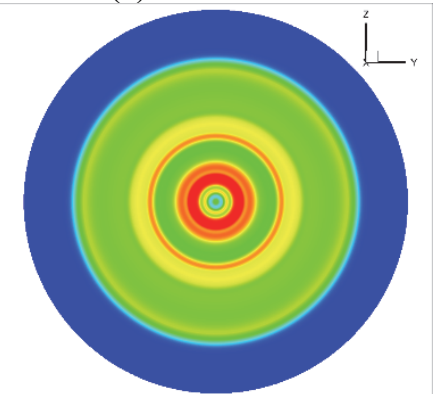

(d) $t=5.11 \mathrm{~ms}$

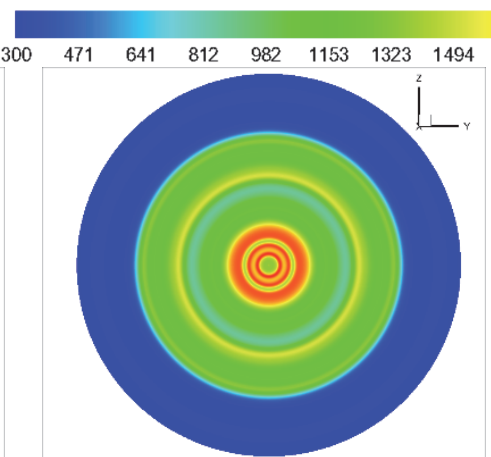

(b) $t=4.65 \mathrm{~ms}$

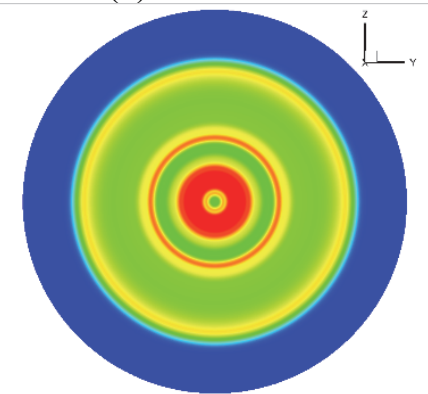

(f) $t=5.25 \mathrm{~ms}$

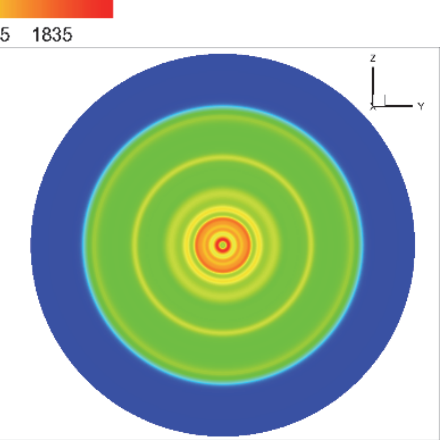

(c) $t=4.83 \mathrm{~ms}$

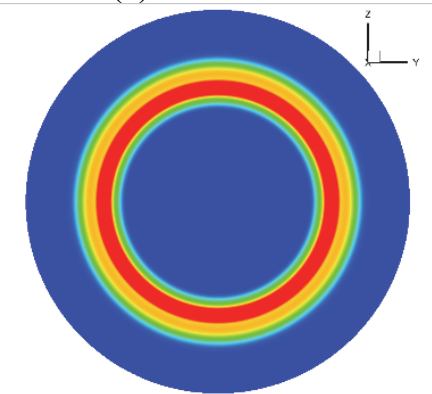

(g) $t=5.33 \mathrm{~ms}$

Figure 15 Cloud map of the temperature of projectile 2 on the $Y O Z$ section at $30 D$ from the right side of the muzzle 


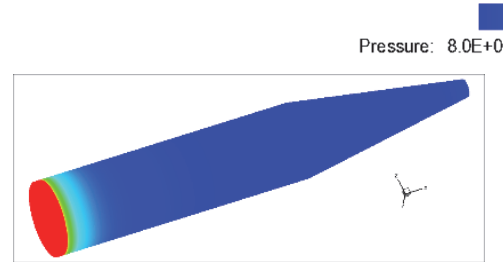

(a) $t=4.54 \mathrm{~ms}$

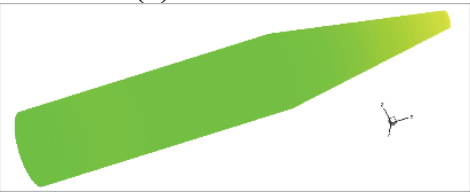

(d) $t=5.11 \mathrm{~ms}$

Figure 16 Distribution of pressure on the surface of projectile 2 in the 3

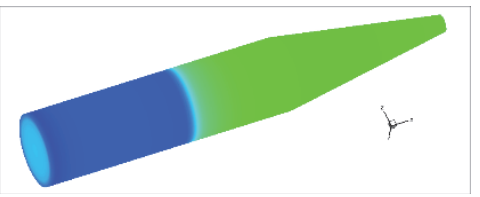

(b) $t=4.65 \mathrm{~ms}$

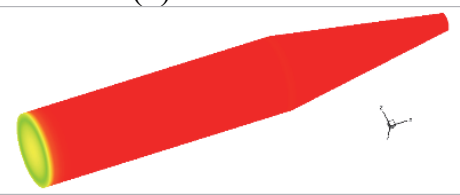

(f) $t=5.25 \mathrm{~ms}$

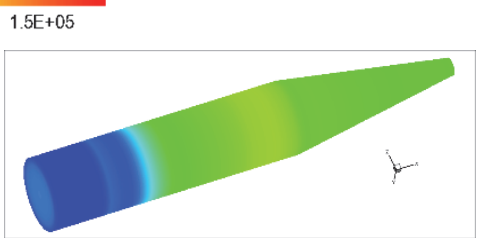

(c) $t=4.83 \mathrm{~ms}$

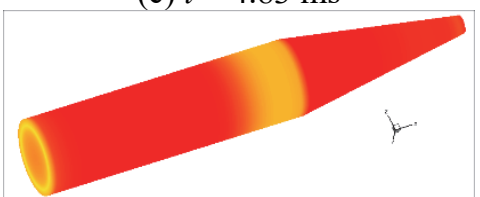

(g) $t=5.33 \mathrm{~ms}$

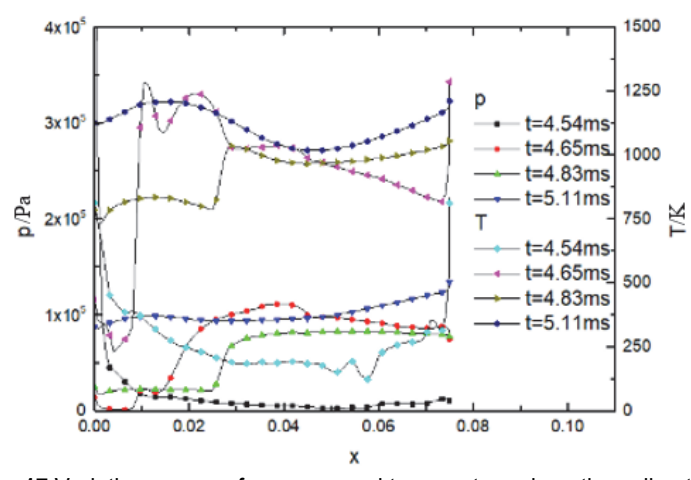

Figure 17 Variation curves of pressure and temperature along the $x$ direction on the surface of projectile 2 in the muzzle flow field at different moments

Fig. 18 and Fig. 19 present the temperature and $\mathrm{OH}$ distribution curves, and the $\mathrm{CO}$ and $\mathrm{CO}_{2}$ distribution curves along the axis of the serial-connected muzzle at different moments, respectively. Obviously, the secondary flame propagated forward along the axis. Fig. 20 compares the flow fields at different moments, when the two projectiles were at unified positions. The temperature changed significantly on the axis, and reached an obviously high level at $t=4.83 \mathrm{~ms}$.

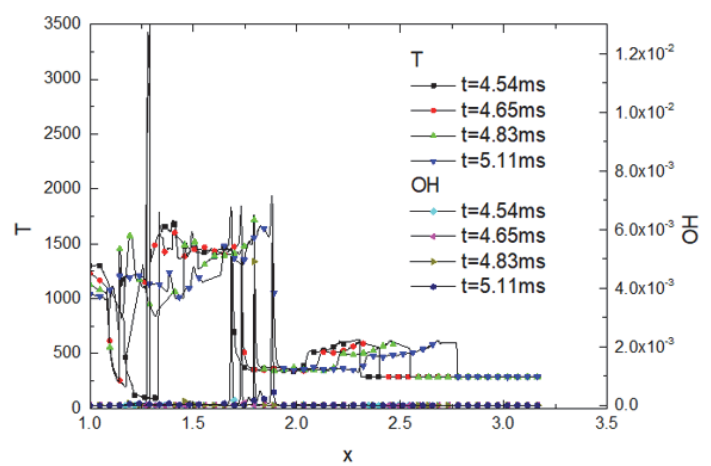

Figure 18 Temperature (left) and $\mathrm{OH}$ (right) distribution curves along the axis of the serial-connected muzzle at different moments

Next, the speedup and parallel efficiency of our parallel algorithm were examined in solving 3D flow problem containing internal moving boundary, using a personal computer with multiple central processing units (CPUs). Two sets of nodes were designed for the simulation: 5,575,322 nodes, and 9,325,832 nodes were deployed in the flow field, respectively. Tab. 2 records the speedup and parallel efficiency at 2,4 , or 8 processes. It can be observed that our parallel algorithm is effective, but not highly efficient, owing to the time consumed to balance computing loads and transmit information in solving the complex 3D problem.

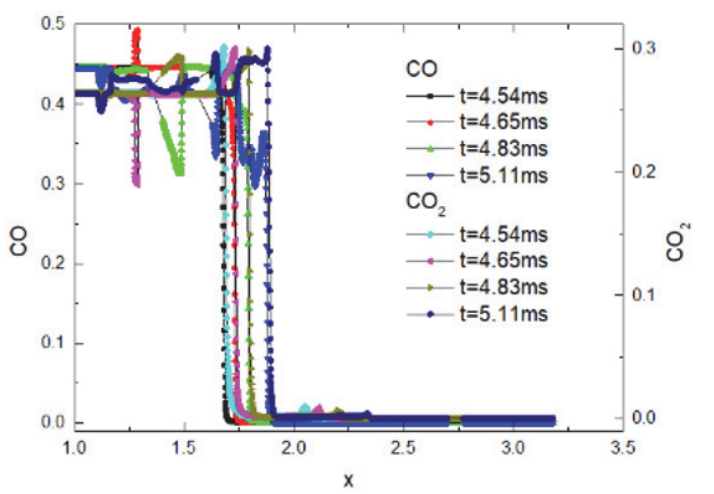

Figure $19 \mathrm{CO}$ and $\mathrm{CO}_{2}$ distribution curves along the axis of the serial-connected muzzle at different moments

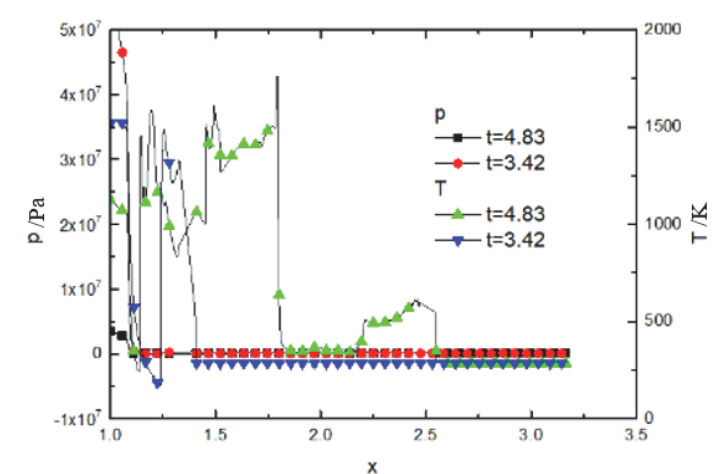

Figure 20 Pressure intensity and temperature distribution curves along the axis of the serial-connected muzzle at $t=3.42 \mathrm{~ms}$ and $4.83 \mathrm{~ms}$

Table 2 Speedup and computing efficiency of parallel algorithm

\begin{tabular}{|c|c|c|c|}
\hline $\begin{array}{c}\text { Number of discrete } \\
\text { nodes }\end{array}$ & $\begin{array}{c}\text { Number of } \\
\text { processes }\end{array}$ & Speedup & $\begin{array}{c}\text { Computing } \\
\text { efficiency }\end{array}$ \\
\hline $5,575,322$ & 2 & 1.709 & $85.5 \%$ \\
\hline $5,575,322$ & 4 & 3.329 & $83.2 \%$ \\
\hline $5,575,322$ & 8 & 6.342 & $79.3 \%$ \\
\hline $9,325,832$ & 2 & 1.766 & $88.3 \%$ \\
\hline $9,325,832$ & 4 & 3.449 & $86.2 \%$ \\
\hline $9,325,832$ & 8 & 6.499 & $81.2 \%$ \\
\hline
\end{tabular}

\section{CONCLUSIONS}

In computational fluid dynamics (CFD), it is a difficulty to compute complex unsteady multi-component $3 \mathrm{D}$ chemical reaction flow, owing to the decoupling of the chemical reaction and the huge computing load. If a 
significant moving boundary exists in the flow field of the chemical reaction, it would be even harder to solve the problem. This paper introduced the gridless method, which eliminates the need of grids, to chemical reaction flow, and sets up a 3D dynamic parallel gridless method. The proposed method can reconstruct the abnormal point cloud near the moving boundary in real time, and adjust the computing load of each parallel zone. Firstly, the flux terms in the ALE control equation were solved by the derived AUFS scheme. The stiff problem of the source term of the chemical reaction was overcome through time splitting. Besides, the flow term and the chemical reaction term were separately calculated multiple times within a variable time step. In addition, local point cloud reconstruction was carried out to reconstruct the abnormal point cloud near the large moving boundary. Then, a number of points was deployed in the cavity through 3D weighted point filling method, and the flow field information was transferred from the original nodes to the new nodes. To reduce the high computing load, the dynamic parallel method was introduced, and the details were given for the parallel computing strategy, zoning strategy, and parallel computing process. During the experimental verification, the multi-component gridless algorithm was proved accurate through single-machine computing of hydrogen combustion reaction in a vessel, and shock-induced combustion with blunt projectile. Next, parallel computing was performed to obtain the $3 \mathrm{D}$ muzzle flow field of prefilled serial-connected projectiles, revealing the evolution of the complex flow field of projectile 2. This paper provides a novel computing method for complex unsteady multi-component 3D chemical reaction flows, containing moving boundary, in future.

\section{Acknowledgements}

This paper is funded by National Natural Science Foundation of China (Grant No.: 51806095); Natural Science Foundation, Jiangsu Province, China (Grant No.: BK20181022); Research Fund of Nanjing Institute of Technology (Grant No.: ZKJ201702).

\section{REFERENCES}

[1] Saghafian, A., Terrapon, V. E., \& Pitsch, H. (2015). An efficient flamelet-based combustion model for compressible flows. Combustion and Flame, 162(3), 652-667. https://doi.org/10.1016/j.combustflame.2014.08.007

[2] Mei, Z. S., Shi, C. Y., Fan, X. L., \& Wang, X. B. (2019). Numerical simulation of hypersonic reentry flow field with gas-phase and surface chemistry models. Materials Today Communications, 22, 100773. https://doi.org/10.1016/j.mtcomm.2019.100773

[3] Santillana, M., Zhang, L., \& Yantosca, R. (2016). Estimating numerical errors due to operator splitting in global atmospheric chemistry models: transport and chemistry. Journal of Computational Physics, 305, 372-386. https://doi.org/10.1016/j.jcp.2015.10.052

[4] Chang, Y., Jia, M., Li, Y., Liu, Y., Xie, M., Wang, H., \& Reitz, R. D. (2015). Development of a skeletal mechanism for diesel surrogate fuel by using a decoupling methodology. Combustion and Flame, 162(10), 3785-3802. https://doi.org/10.1016/j.combustflame.2015.07.016
[5] Chen, J. S., Roque, C., Pan, C., \& Button, S. T. (1998). Analysis of metal forming process based on meshless method. Journal of Materials Processing Technology, 80-81, 642-646. https://doi.org/10.1016/S0924-0136(98)00171-X

[6] Wang, L., Xu H. Q., Wu W., \& Xue R. (2016). A novel gridless adaptive method for simulating unsteady flows. Journal of Aerodynamics, 4, 497-502. https://doi.org/10.1142/9789813145603_0137

[7] Yanenko, N. N. \& Holt, M. (1971). The Method of Fractional Steps. Springer-Verlag. https://doi.org/10.1007/978-3-642-65108-3

[8] Trotter, H. F. (1959). On the product of semi-groups of operators. Proceedings of the American Mathematical Society, 10(4), 545-551. https://doi.org/10.2307/2033649

[9] Strang, G. (1968). On the construction and comparison of difference schemes. Siam Journal on Numerical Analysis, 5(3), 506-517. https://doi.org/10.1137/0705041

[10] Shuen, J. S. \& Yoon, S. (1989). Numerical study of chemically reacting flows using a lower-upper symmetric successive overrelaxation scheme. AIAA Journal, 27(12), 1752-1760. https://doi.org/10.2514/3.10331

[11] Liu, J., Liu, Y., \& Zhou, S. (2010). Simulation of shock induced combustion based on a novel uncoupled method. LixueXuebao/Chinese Journal of Theoretical and Applied Mechanics, 42(3), 572-578.

[12] Liu, J. (2003). Numerical study on chemical mechanism in supersonic h2/air mixture gas flow. TuijinJishu/Journal of Propulsion Technology, 24(1), 67-70.

[13] Liu, Y., Liu, J., Tang, L., \& Cui, X. (2015). A novel uncoupled algorithm for solving chemical nonequilibrium flows. LixueXuebao/Chinese Journal of Theoretical and Applied Mechanics, 47(1), 82-94.

[14] Ziegler, J. L., Deiterding, R., Shepherd, J. E., \& Pullin, D. I. (2011). An adaptive high-order hybrid scheme for compressive, viscous flows with detailed chemistry. Journal of Computational Physics, 230(20), 7598-7630. https://doi.org/10.1016/j.jcp.2011.06.016

[15] Lv, Y., \& Ihme, M. (2014). Discontinuous galerkin method for multicomponent chemically reacting flows and combustion. Journal of Computational Physics, 270(1), 105137. https://doi.org/10.1016/j.jcp.2014.03.029

[16] Duarte, M., Descombes, S.,Tenaud, C.,Candel, S., \& Massot, M. (2013). Time-space adaptive numerical methods for the simulation of combustion fronts. Combustion \& Flame, 160(6), 1083-1101. https://doi.org/10.1016/j.combustflame.2013.01.013

[17] Gou, X., Sun, W., Chen, Z., \& Ju, Y. (2010). A dynamic multi-timescale method for combustion modeling with detailed and reduced chemical kinetic mechanisms. Combustion \& Flame, 157(6), 1111-1121. https://doi.org/10.1016/j.combustflame.2010.02.020

[18] Sun, W., Gou, X., El-Asrag, H. A., Chen, Z., \& Ju, Y. (2015). Multi-timescale and correlated dynamic adaptive chemistry modeling of ignition and flame propagation using a real jet fuel surrogate model. Combustion \& Flame, 162(4), 15301539. https://doi.org/10.1016/j.combustflame.2014.11.017

[19] Zhuo, C. F., Xiao-Song, W. U., Feng, F., \& Deng, H. Y. (2014). Numerical simulation of chemical non-equilibrium flow based on gridless algorithm. TuijinJishu/Journal of Propulsion Technology, 35(2), 244-250.

[20] Huh, J. Y., Kim, K. H., \& Jung, S. Y. (2014). Meshless method for simulation of compressible reacting flow. Cities \& Disaster Reduction.

[21] Bayona, V., Sánchez-Sanz, M., Fernández-Tarrazo, E., \& Kindelan, M. (2021). Micro-combustion modelling with RBF-FD: a high-order meshfree method for reactive flows in complex geometries. Applied Mathematical Modelling, 94, 635-655. https://doi.org/10.1016/j.apm.2021.01.032 
[22] Sussman, M. (1994). A computational study of unsteady shock-induced combustion of hydrogen-air mixtures. AIAA Paper. https://doi.org/10.2514/6.1994-3101

[23] Oktay, E., Akay, H. U., \& Merttopcuoglu, O. (2011). Parallelized structural topology optimization and CFD coupling for design of aircraft wing structures. Computers \& Fluids, 49(1), 141-145. https://doi.org/10.1016/j.compfluid.2011.05.005

[24] Wang, L., Xue, R., Cai, N., Wu, W., Niu, M. M., \& Zhang, D. L. (2020). Application of least squares meshless method in multi-component high-speed non-equilibrium reaction jet. International Journal of Design \& Nature and Ecodynamics, 15(4), 507-514. https://doi.org/10.18280/ijdne.150407

[25] Pozzetti, G., Jasak, H., Besseron, X., Rousset, A., \& Peters, B. (2019). A parallel dual-grid multiscale approach to cfddem couplings. Journal of computational physics, 378, 708722. https://doi.org/10.1016/j.jcp.2018.11.030

[26] Singh, I. V. (2004). Parallel implementation of the efg method for heat transfer and fluid flow problems. Computational Mechanics, 34(6), 453-463. https://doi.org/10.1007/s00466-004-0590-0

[27] Ma, Z. H., Wang, H., \& Pu, S. H. (2015). A parallel meshless dynamic cloud method on graphic processing units for unsteady compressible flows past moving boundaries. Computer Methods in Applied Mechanics and Engineering, 285, 146-165. https://doi.org/10.1016/j.cma.2014.11.010

[28] Wang, H., Chen, H. Q., \& Periaux, J. (2010). A study of gridless method with dynamic clouds of points for solving unsteady cfd problems in aerodynamics. International Journal for Numerical Methods in Fluids, 64(1), 98-118. https://doi.org/10.1002/fld.2145

[29] Zhang, J. L., Chen, H. Q., Xu, S. G., \& Gao, H. Q. (2020). A novel gpu-parallelized meshless method for solving compressible turbulent flows. Computers \& Mathematics with Applications, 80(12), 2738-2763. https://doi.org/10.1016/j.camwa.2020.08.030

[30] Barbosa, M., Telles, J., Santiago, J., Junior, E., \& Costa, E. (2021). A parallel implementation strategy for meshless methods based on the functional programming paradigm. Advances in Engineering Software, (151), 102926. https://doi.org/10.1016/j.advengsoft.2020.102926

[31] Sun, M. \& Takayama, K. (2003). An artificially upstream flux vector splitting scheme for the euler equations. Journal of Computational Physics, 189(1), 305-329. https://doi.org/10.1016/S0021-9991(03)00212-2

[32] Soetrisno M. \& Imlay S. (1991). Simulation of the Flow Field of a Ram Accelerator. AIAA Paper. https://doi.org/10.2514/6.1991-1915

\section{Contact information:}

\section{Liang WANG}

(Corresponding author)

School of Energy and Power Engineering,

Nanjing Institute of Technology,

Nanjing 211167, China

E-mail: wl_njit@njit.edu.cn

\section{Ning CAI}

School of Energy and Power Engineering, Nanjing Institute of Technology,

Nanjing 211167, China

E-mail: cnnjit@126.com

\section{Rui XUE}

School of Energy and Power Engineering, Nanjing Institute of Technology,

Nanjing 211167, China

E-mail: xuerui@njit.edu.cn

\section{Xiaobo CUI}

School of Energy and Power Engineering,

Nanjing Institute of Technology,

Nanjing 211167, China

E-mail: xiaobo@njit.edu.cn 